\title{
Similarity Scaling Over a Steep Alpine Slope
}

\author{
Daniel F. Nadeau • Eric R. Pardyjak • \\ Chad W. Higgins - Marc B. Parlange
}

Received: 27 July 2012 / Accepted: 22 November 2012

(C) Springer Science+Business Media Dordrecht 2012

\begin{abstract}
In this study, we investigate the validity of similarity scaling over a steep mountain slope $\left(30-41^{\circ}\right)$. The results are based on eddy-covariance data collected during the Slope Experiment near La Fouly (SELF-2010); a field campaign conducted in a narrow valley of the Swiss Alps during summer 2010. The turbulent fluxes of heat and momentum are found to vary significantly with height in the first few metres above the inclined surface. These variations exceed by an order of magnitude the well-accepted maximum $10 \%$ required for the applicability of Monin-Obukhov similarity theory in the surface layer. This could be due to a surface layer that is too thin to be detected or to the presence of advective fluxes. It is shown that local scaling can be a useful tool in these cases when surface-layer theory breaks down. Under convective conditions and after removing the effects of self-correlation, the normalized standard deviations of slope-normal wind velocity, temperature and humidity scale relatively well with $z / \Lambda$, where $z$ is the measurement height and $\Lambda(z)$ the local Obukhov length. However, the horizontal velocity fluctuations are not correlated with $z / \Lambda$ under all stability regimes. The non-dimensional gradients of wind velocity and temperature are also investigated. For those, the local scaling appears inappropriate, particularly at night when shallow drainage flows prevail and lead to negative wind-speed gradients close to the surface.
\end{abstract}

D. F. Nadeau - M. B. Parlange

School of Architecture, Civil and Environmental Engineering, École Polytechnique Fédérale de Lausanne, Station 2, 1015 Lausanne, Switzerland

E. R. Pardyjak

Department of Mechanical Engineering, University of Utah, Salt Lake City, UT 84112, USA

C. W. Higgins

Department of Biological and Ecological Engineering, Oregon State University, Corvallis, OR 97331, USA

Present Address:

D. F. Nadeau $(\varangle)$

Institut National de la Recherche Scientifique, Centre Eau, Terre et Environnement, Quebec, QC, Canada e-mail: daniel.nadeau.1@gmail.com 
Keywords Drainage flow · Downslope flow · Flux divergence ·

Flux-gradient relationships · Flux-variance relationships · Local similarity ·

Mountain winds $\cdot$ Surface layer

\section{Introduction}

A number of the world's mountainous areas, such as the European Alps, are currently facing significant urban growth (Perlik et al. 2001). To ensure the security and economic prosperity of these populations, meteorologists are exhorted to provide accurate weather forecasts, which rely heavily on the understanding of the small-scale physical processes near the complex alpine surface. Research efforts on the alpine atmospheric boundary layer have been substantial in the past 15-20 years (e.g. Grubisic et al. 2008), yet most of the work has focused on idealized sites, and thus not always representative of a realistic mountainous landscape with steep slopes and meandering valleys.

The main features of mountain flows are relatively well understood. For instance, alpine winds can be divided into two categories: terrain-forced flows and diurnal flows (Whiteman 2000). Terrain-forced flows such as channeled winds are driven by large-scale synoptic pressure gradients. Diurnal winds are driven by the uneven heating of the atmosphere inside the valley, which results in up-valley/upslope flow during the day and down-valley/downslope flow at night. In most situations, synoptically- and thermally-driven flows overlap.

To simulate these flows, numerical weather prediction models use similarity scaling, but little is known about its applicability over such highly complex terrain. The use of scaling relationships to study turbulence in the atmospheric boundary layer (ABL) has been common practice for decades (Holtslag and Nieuwstadt 1986). The idea behind scaling is that the physical characteristics of the ABL can be expressed as a function of a few non-dimensional parameters. The first studies that tested similarity scaling focused on flat and ideal terrain (e.g. Businger et al. 1971), but recent work has shown that boundary-layer turbulence in a complex alpine valley still displayed reproducible and characteristic patterns that would allow the use of similarity theory (Rotach and Zardi 2007).

Among the various classes of similarity scaling, Monin-Obukhov similarity theory (MOST), derived for the atmospheric surface layer (Monin and Obukhov 1954), is among the most widely used. MOST applies over flat and homogeneous terrain, for quasi-stationary atmospheric conditions and when rotational effects in the flow are negligible. While it is obvious that these criteria are rarely met, MOST remains a relatively robust and widely used tool to relate turbulent fluxes to mean gradients, variances or spectral characteristics (Foken 2006). MOST applies in the inertial sublayer of the surface layer, in which the turbulent fluxes are nearly constant with height (variations of $10 \%$ at most).

The applicability of MOST has been extensively studied over a wide range of landscapes, from equatorial areas (Yusup et al. 2008), to deserts (Tamagawa 1996), forests (Rannik 1998), wetlands (Marques et al. 2008), alpine valley floors (de Franceschi et al. 2009), heterogeneous surfaces (Andreas et al. 1998), lakes (Drennan et al. 1999), rugged hilly landscapes (Brutsaert and Kustas 1987), and urban areas (Nordbo et al. 2012).

There are some well-known cases when surface-layer scaling is not expected to apply. For stably stratified flows, for instance, internal gravity waves, Kelvin-Helmholtz shear instabilities, density currents, and low-level jets can all signify a departure from MOST (Cheng et al. 2005). For a strongly unstable regime, free convective scaling is more relevant than MOST. Over complex inhomogeneous surfaces, the presence of internal boundary layers and 
local advection leads to the non-negligible divergence of the turbulent fluxes (Högström et al. 1982), and thus MOST is not expected to apply.

Local similarity scaling can be a useful approach to study the physical properties of such complex flows. Local similarity applies at specific locations in the boundary layer, unlike MOST, which applies inside the constant-flux layer (Shao and Hacker 1990). According to the local scaling hypothesis, the turbulent quantities are a function of the local fluxes evaluated at a height $z$. Local similarity holds when boundary-layer turbulence is not in equilibrium with the surface fluxes. For instance, local scaling can be applied in the upper regions of the nocturnal stable boundary layer, where the eddies are small and disconnected with the surface (Mahrt 1999). In the local similarity framework, $z$ is a highly significant parameter.

Local scaling has been traditionally applied above the surface layer, following the pioneering work of Nieuwstadt (1984) and Sorbjan (1988), who used it for stable and convective boundary layers, respectively. For instance, Wood et al. (2010) recently detailed a successful use of local scaling with turbulence measurements collected at $190 \mathrm{~m}$ above the city of London, UK, similar to what Al-Jiboori et al. (2002) had shown with observations from a 325-m meteorological mast in Beijing, China. Local scaling has also been successfully demonstrated with airborne measurements over coastal zones (see Shao and Hacker 1990; Brooks and Rogers 2000).

Very little work has been carried out for similarity scaling over steep slopes, where there is also a considerable lack of observations. Grisogono et al. (2007) recently provided a theoretical analysis on the reliability of MOST for drainage flows over small slopes and proposed a modified Obukhov length that incorporates the height of the katabatic jet. Park and Park (2006) studied surface-layer turbulence characteristics over a university campus surrounded by sloping terrain, but the maximum slopes were still relatively small $\left(14^{\circ}\right)$. Rotach et al. (2008) presented a few results of scaled vertical velocity fluctuations collected over a $30^{\circ}$ alpine slope, highlighting the fact that similarity of near-surface variables in complex alpine terrain was a "necessary domain of future research". Martins et al. (2009) probably provided the most complete overview on the subject, with the help of field measurements on the side of a nearly $40^{\circ}$ slope. They found that the variance of the vertical velocity and the temperature obeyed MOST for all stability conditions and wind direction, while horizontal velocity followed MOST only for a small range of stabilities. The latter finding is consistent with previous studies conducted over flat terrain for which the horizontal velocity variances scaled with boundary-layer depth and the convective velocity scale (e.g. Marques et al. 2008). The results of Martins et al. (2009) were based on a single level of turbulence measurements, and so could not confirm whether their observations were collected in a constant-flux layer, thus denying a rigorous evaluation of MOST.

The objective of this paper is to examine the applicability of similarity scaling over a steep alpine slope, on the basis of multi-level turbulence observations. We start our analysis by assessing the height dependence of the turbulent fluxes, and show that surface-layer theory is not valid at our site. Using a local scaling framework, we then investigate the applicability of flux-variance relationships for wind velocity components, temperature and humidity, and flux-gradient relationships for wind speed and temperature.

\section{Theoretical Background}

According to local similarity, the mean dimensionless wind shear should solely be a function of the local stability parameter $\zeta=z / \Lambda$ : 


$$
\left(\frac{\kappa z}{u_{* l}}\right) \frac{\partial \bar{u}}{\partial z}=\phi_{m}(\zeta),
$$

where $z$ is the measurement height, $\Lambda$ is the local Obukhov length and varies with height, $\kappa$ is the Von Kármán constant $(=0.4), u_{* l}$ is the local friction velocity and $u$ is the horizontal wind speed. Here overbars are used for time averaging and primes for fluctuating quantities. By definition, $\phi_{m}(0)=1$ in the neutral regime. The local Obukhov length is representative of the ratio of mechanical to thermal production/destruction of turbulence, and is expressed as

$$
\Lambda(z)=-\frac{u_{* l}^{3} \overline{\theta_{v}}}{\kappa g \overline{w^{\prime} \theta_{v}^{\prime}}},
$$

where $\theta_{v}$ is a characteristic virtual potential air temperature, $g$ is the gravitational acceleration and $\overline{w^{\prime} \theta_{v}^{\prime}}$ is the buoyancy flux. The local friction velocity $u_{* l}$ is based on the local Reynolds stress, such that

$$
u_{* l}=\left({\overline{u^{\prime} w^{\prime}}}^{2}+{\overline{v^{\prime} w^{\prime}}}^{2}\right)^{1 / 4}
$$

where $u$ and $v$ are the horizontal components of wind speed. The equivalent of (1) for potential temperature $\theta$ is

$$
\left(\frac{\kappa z}{\theta_{* l}}\right) \frac{\partial \bar{\theta}}{\partial z}=\phi_{h}(\zeta),
$$

where $\theta_{* l}=-\overline{w^{\prime} \theta^{\prime}} / u_{* l}$ is the local temperature scale, while for humidity one finds

$$
\left(\frac{\kappa z}{q_{* l}}\right) \frac{\partial \bar{q}}{\partial z}=\phi_{q}(\zeta)
$$

with $q_{* l}=-\overline{w^{\prime} q^{\prime}} / u_{* l}, q$ being the specific humidity of air. A detailed description of the universal functions $\phi_{m}, \phi_{h}$ and $\phi_{q}$ can be found in Brutsaert (2005).

Similar relationships also hold for scaled standard deviations:

$$
\begin{aligned}
\frac{\sigma_{i}}{u_{* l}} & =\Phi_{i}(\zeta), \\
\frac{\sigma_{\theta}}{\left|\theta_{* l}\right|} & =\Phi_{\theta}(\zeta), \\
\frac{\sigma_{q}}{\left|q_{* l}\right|} & =\Phi_{q}(\zeta),
\end{aligned}
$$

where $\sigma_{i}$ is the standard deviation of a velocity component $(i=u, v, w), \sigma_{\theta}$ is the standard deviation of temperature, and $\sigma_{q}$ is the standard deviation of humidity. The formulation of the $\Phi$ functions varies between studies, and with atmospheric stability, and an exhaustive review can be found in de Franceschi et al. (2009). A widely accepted form for velocity fluctuations in the MOST framework is

$$
\Phi_{i}=a_{i}\left(1+b_{i}|\zeta|\right)^{1 / 3}
$$

in which $a_{i}$ and $b_{i}$ are found experimentally. For temperature and humidity, one typically finds

$$
\Phi_{\theta, q}= \begin{cases}a_{\theta, q}\left(1-b_{\theta, q} \zeta\right)^{-1 / 3} & \text { for } \zeta<0, \\ c_{\theta, q}\left(1+d_{\theta, q} \zeta\right)^{-1} & \text { for } \zeta>0 .\end{cases}
$$

where $a_{\theta, q}, b_{\theta_{q}}, c_{\theta_{q}}$ and $d_{\theta, q}$ are also empirically determined. 


\section{Methods}

\subsection{Experimental Site}

The research site of the Slope Experiment near La Fouly (SELF-2010) is located on a steep west-facing slope of Val Ferret in the Swiss Alps $\left(45^{\circ} 54^{\prime} 7^{\prime \prime} \mathrm{N}, 7^{\circ} 7^{\prime} 24^{\prime \prime} \mathrm{E} ; \approx 2,000 \mathrm{~m}\right.$ above sea level, a.s.1.). The Swiss Val Ferret is a narrow alpine valley (width $\approx 5 \mathrm{~km}$, depth $\approx 1 \mathrm{~km}$ ) bordered to the south by Italy, and to the west by France. The experimental site is located in a curve of the main valley axis, where the orientation transitions from south-east-north-west to north-south (Fig. 1). The vegetation is typical of an alpine region, with grass and some shrubs up to approximately $2,500 \mathrm{~m}$ a.s.l.

In this study, we assume that the zero-plane displacement height $d_{0} \approx 0$. On the basis of experimental data, the logarithmic mean of the aerodynamic roughness length $z_{0}$ is found to give $z_{0} \approx 0.02 \mathrm{~m}$ for the two main wind sectors (see Fig. 2).
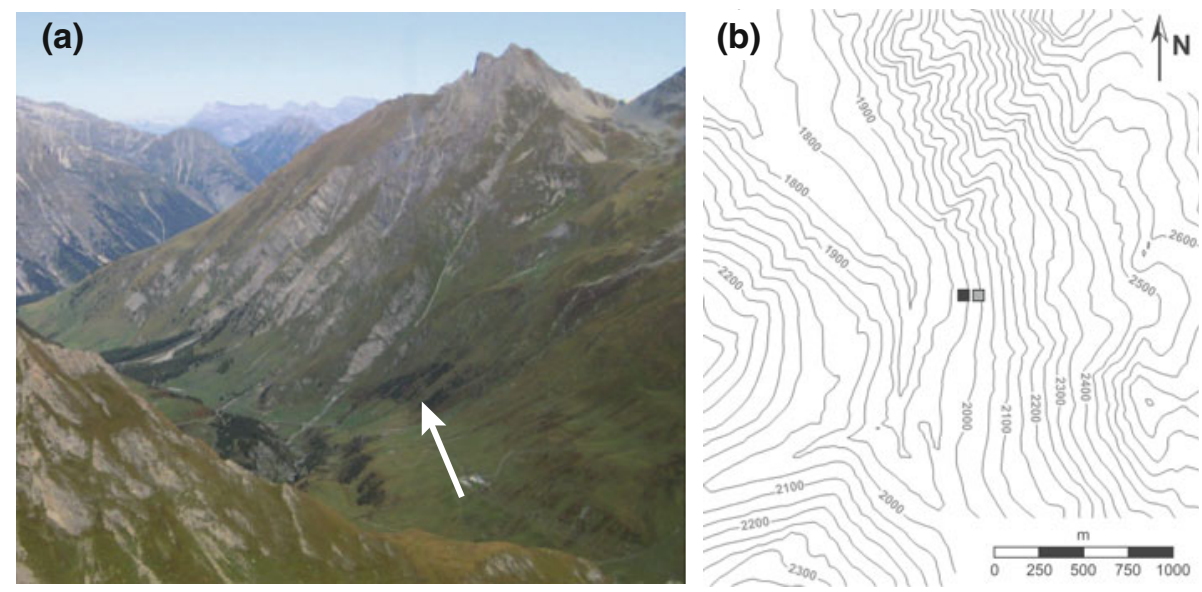

Fig. 1 a Aerial photograph of the field site (white arrow) looking to the north taken on 21 September 2010 at 1115 CET (Central European Time) in Val Ferret, Switzerland. b Topographic map with contours lines each $50 \mathrm{~m}$. The locations of the towers T1 and T2 are indicated by a black and grey square, respectively

(a)

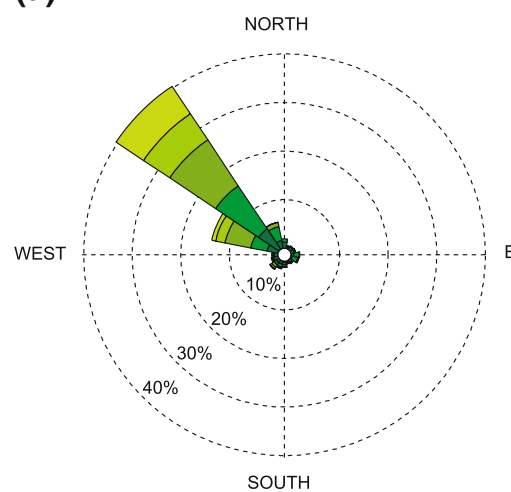

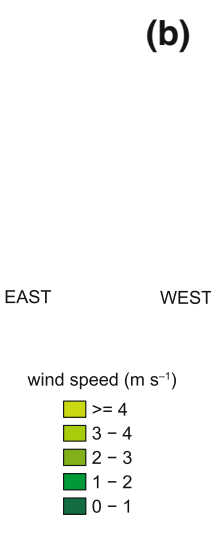

(b)

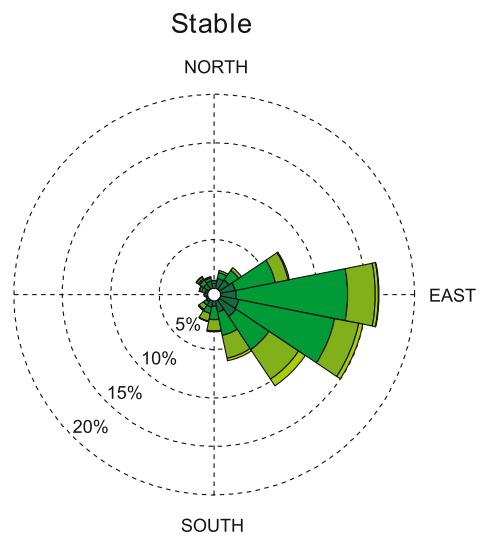

Fig. 2 Wind rose at site T1 a for convective conditions $(\zeta<0)$ and $\mathbf{b}$ for stable conditions $(\zeta>0)$. Note the dominant up-valley/upslope winds under unstable conditions (daytime) and the downslope winds under stable conditions (nighttime) 
Table 1 Location and topographic characteristics of the two sites

\begin{tabular}{llllll}
\hline Site & Latitude & Longitude & $\begin{array}{l}\text { Elevation } \\
(\mathrm{m} \text { a.s.1. })\end{array}$ & $\begin{array}{l}\text { Slope } \\
\text { inclination }\left(^{\circ}\right)\end{array}$ & $\begin{array}{l}\text { Slope } \\
\text { aspect }\end{array}$ \\
\hline $\mathrm{T} 1$ & $45^{\circ} 54^{\prime} 7^{\prime} \mathrm{N}$ & $7^{\circ} 7^{\prime} 23^{\prime \prime} \mathrm{E}$ & 1,939 & 30 & West \\
$\mathrm{T} 2$ & $45^{\circ} 54^{\prime} 7^{\prime \prime} \mathrm{N}$ & $7^{\circ} 7^{\prime} 26^{\prime \prime} \mathrm{E}$ & 1,988 & 41 & West \\
\hline
\end{tabular}

\subsection{Data}

The data used herein were collected from 7 July to 29 September 2010. Rainy days were excluded from the analysis, yielding a total dataset of 36 days, including nine with nearly perfect clear-sky conditions.

The measurement set-up is fully described in Nadeau et al. (2012), though for completeness, we repeat the most relevant details here. Among several other meteorological stations, two eddy-covariance measurement systems (stations T1 and T2) were deployed along an experimental transect of a steep alpine slope (Table 1; Fig. 1b). Station T1 had two CSAT3 ultrasonic anemometers (Campbell Scientific, USA) facing the upslope and downslope directions ( 2.5 and $2.0 \mathrm{~m}$ above the surface ${ }^{1}$ ) as well as an open path infrared fast-response $\mathrm{CO}_{2}-\mathrm{H}_{2} \mathrm{O}$ analyzer (Model 7500, Licor, USA) installed at $2.0 \mathrm{~m}$ above the surface. At station $\mathrm{T} 2$, at three heights $(1.5,4.7$ and $5.9 \mathrm{~m})$, turbulence measurements of wind speed and sonic temperature were collected by CSAT3 ultrasonic anemometers facing north. All sonic anemometers were installed in a slope-normal configuration as suggested by Geissbuhler et al. (2000) for non-flat terrain.

The data sampling frequency was set to $10 \mathrm{~Hz}$ for the turbulence sensors, with turbulence statistics obtained with the eddy-covariance method using averaging periods of 15 min. This averaging time scale was selected based on analyses of 'ogive' functions. After being linearly detrended, the turbulence measurements were rotated into a mean streamline plane obtained by regressing a collection of data segments over a long time period (a few weeks). A regression was obtained for each of four wind sectors corresponding to the upslope, up-valley, downslope and down-valley directions. This "directional planar fit regression" (see Nadeau et al. 2012) is an adaptation of the method described in Wilczak et al. (2001). Each data segment was subsequently aligned with the mean flow to obtain $\bar{v}=0$.

In this study, we assumed that the sonic temperature $T_{\mathrm{s}}=T(1+0.51 q)$, where $T$ is the air temperature, was equal to the virtual potential temperature $\theta_{v}$, given that the measurements are collected a few metres above the surface. All data segments with wind direction from a $120^{\circ}$ sector behind the instruments were excluded from the analysis. We assumed that all measurements were collected above the roughness sublayer, given that the vegetation at the surface was relatively short $(\approx 0.3 \mathrm{~m}$ tall $)$.

We used a slope coordinate system, with $x$ aligned with the flow and $z$ normal to the slope. We rejected data segments with the magnitude of the sensible heat flux $|H|<10 \mathrm{~W} \mathrm{~m}^{-2}$ as recommended by de Franceschi et al. (2009) to exclude transition periods from the analysis, and thus to prevent indeterminate ratios such as $\sigma_{\theta} / \theta_{* l}$ in the near-neutral regime.

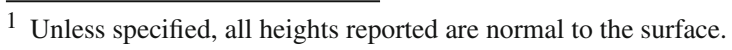




\section{Results and Discussion}

\subsection{Brief Climatology}

During the experimental period, the turbulent fluxes of sensible and latent heat usually peaked around 1400-1500 Central European Time (CET) when the incoming shortwave radiation normal to the slope was a maximum $\left(\approx 850-1,000 \mathrm{~W} \mathrm{~m}^{-2}\right)$. The surface temperatures varied between -1 and $26^{\circ} \mathrm{C}$, and the air temperatures between 1 and $23^{\circ} \mathrm{C}$. The mean specific humidity was $7.7 \mathrm{~g} \mathrm{~kg}^{-1}$, and the mean wind speed was $1.6 \mathrm{~m} \mathrm{~s}^{-1}$.

The wind-direction frequency distribution is plotted in Fig. 2. Under convective conditions, the winds were almost exclusively up-valley/upslope (Fig. 2a). The presence of an upslope component was partially induced by the curvature of the valley topography (Fig. 1b). Up-valley winds were expected when thermally-driven circulations prevailed during the day. Figure $2 \mathrm{a}$ also shows that synoptic forcing either acted to strengthen the thermally-driven up-valley flow or was too weak to force a down-valley flow during the day. At night, there was a greater spread in wind directions. The lowest layers of the atmosphere were possibly disconnected from aloft (Nadeau et al. 2012) and the winds were less channeled by the valley topography. Downslope and down-valley winds were observed in most cases. Overall the winds were lighter at night.

\subsection{Variation of Turbulent Fluxes with Height}

A majority of the studies on the applicability of similarity scaling have not assessed whether their measurements were collected in a region where turbulent fluxes were constant with height. In most cases, this is because only one level of turbulence measurements was available. Mahrt (1999) argued that in these cases, it is not clear which aspects of MOST or local similarity are being evaluated.

Figures 3 and 5 present box plots of buoyancy and momentum fluxes at three different heights. The data segments were divided into unstable and stable conditions by evaluating the stability parameter $\zeta$ at the lowest observational level.

In Fig. 3, we note that the magnitude of the buoyancy flux tends to be greater at $1.5 \mathrm{~m}$ above the surface. Note that there are cases for which the buoyancy fluxes at different levels are of different signs, although these might be random flux errors (Lenschow et al. 1994). Judging by the median values, $\left|\overline{w^{\prime} \theta_{v}^{\prime}}\right|$ varies by more than $100 \%$ over just a few metres, probably because
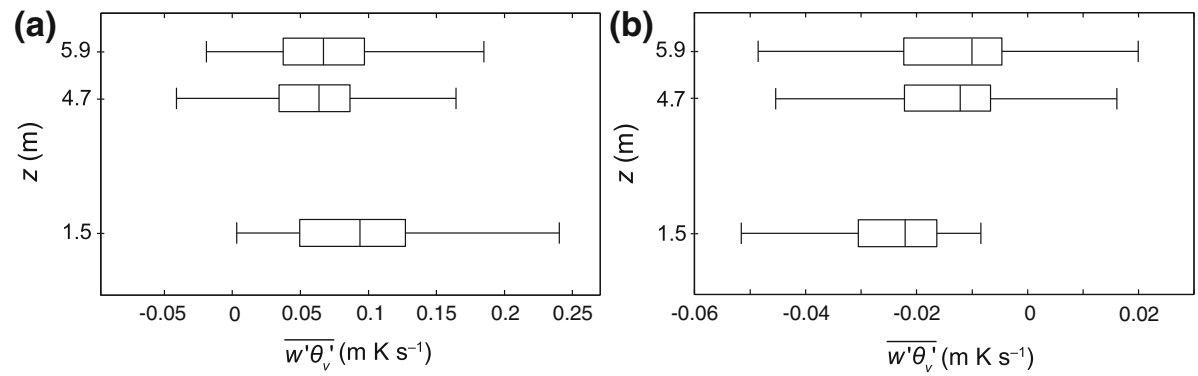

Fig. 3 Box plots of buoyancy flux variations with height for data segments with $\mathbf{a} \zeta<0$ and $\mathbf{b} \zeta>0$ at the lowest observational level of station T2 (1.5 m normal to the surface). The mark inside the box represents the median, the edges are the lower and upper quartiles, and the whiskers extends approximately to \pm 2.7 times the standard deviation of the data. Outliers were excluded from the plot 

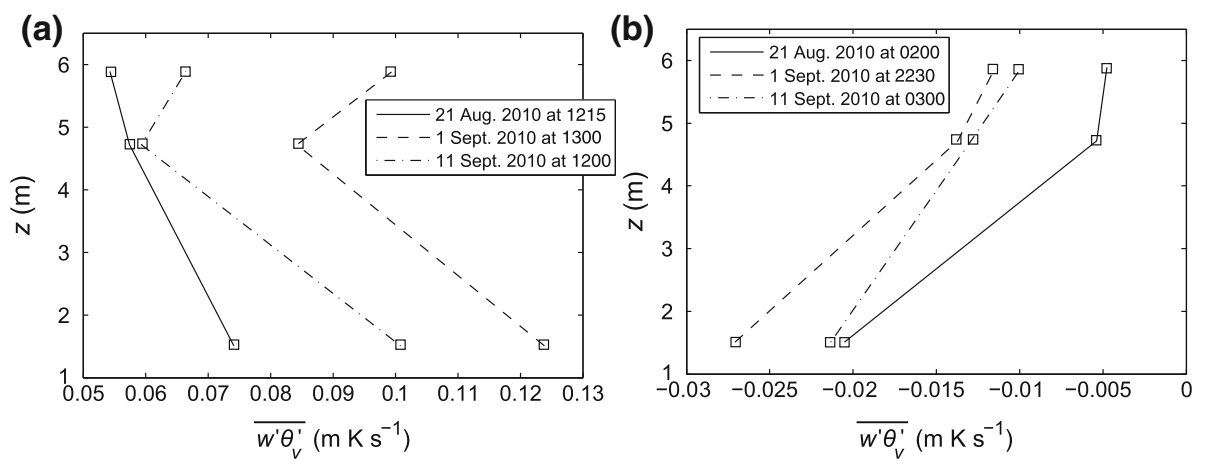

Fig. 4 Sample 15-min buoyancy flux variations with height for data segments with $\mathbf{a} \zeta<0$ and $\mathbf{b} \zeta>0$ at the lowest observational level of station T2 (1.5 m normal to the surface). Central European times are used
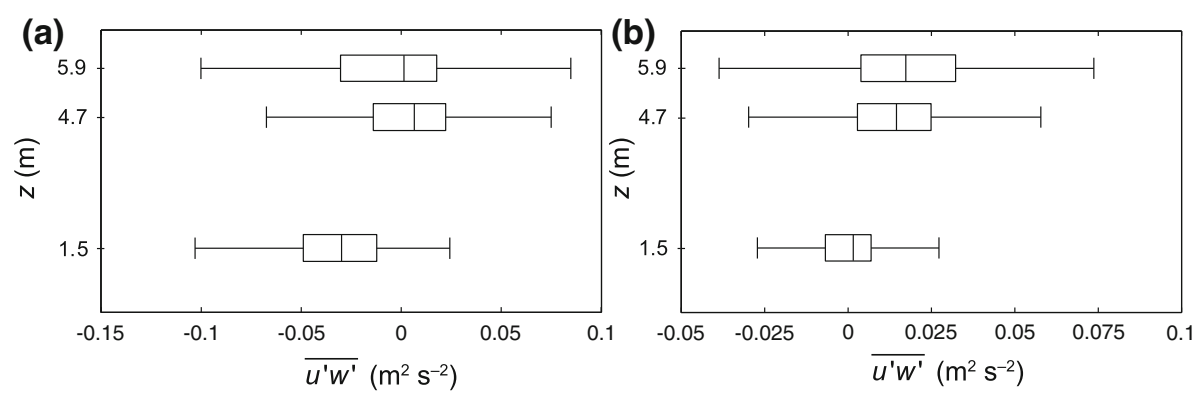

Fig. 5 Box plots of momentum flux variations with height for data segments with $\mathbf{a} \zeta<0$ and $\mathbf{b} \zeta>0$ at the lowest observation level of station T2 (1.5 m normal to the surface). The mark inside the box represents the median, the edges are the lower and upper quartiles, and the whiskers extends approximately to \pm 2.7 times the standard deviation of the data. Outliers were excluded from the plot

of the advective fluxes associated with the thermal circulations. This height dependence of $\overline{w^{\prime} \theta_{v}^{\prime}}$ is incompatible with MOST, which requires a nearly constant-flux layer. Figure 4 shows a few sample profiles of the buoyancy flux highlighting the strong variations with height. Under unstable conditions, the mean buoyancy flux divergence evaluated with a centered finite difference at $3.7 \mathrm{~m}$ is $-0.0053 \mathrm{~K} \mathrm{~s}^{-1}\left(\approx-19 \mathrm{~K} \mathrm{~h}^{-1}\right)$, and the standard deviation is $0.0053 \mathrm{~K} \mathrm{~s}^{-1}$. Under stable conditions, these values are respectively $0.0015 \mathrm{~K} \mathrm{~s}^{-1}\left(\approx 5 \mathrm{~K} \mathrm{~h}^{-1}\right)$ and $0.0022 \mathrm{~K} \mathrm{~s}^{-1}$.

At the two highest observational levels, the median values of $\overline{u^{\prime} w^{\prime}} \gtrsim 0$, which suggests that $\partial \bar{u} / \partial z \lesssim 0$ during the day (Fig. 5). Nadeau et al. (2012) noted that, prior to the evening transition on clear-sky days, the wind shear was particularly small. They hypothesized that the weak shear may have been a result of the "impinging" up-valley flow since the experimental site is located in a curve of the valley axis. Notice that, for most of the segments, $\overline{u^{\prime} w^{\prime}}>0$ at night. This is due to the formation of a shallow drainage flow (or 'skin flow'), with maximum velocities below $1.5 \mathrm{~m}$, leading to $\partial \bar{u} / \partial z<0$ in the observational layer. There is remarkable scatter in the momentum flux values at the two highest measurement levels, but overall greater magnitudes of $\overline{u^{\prime} w^{\prime}}$ are observed at the highest levels at night. This might be detached turbulence induced by the shear in the upper part of the drainage flow (Mahrt 1999). Figure 6 shows a few sample profiles of momentum flux highlighting the strong variations with height. Under unstable conditions, the mean momentum flux divergence evaluated with a 

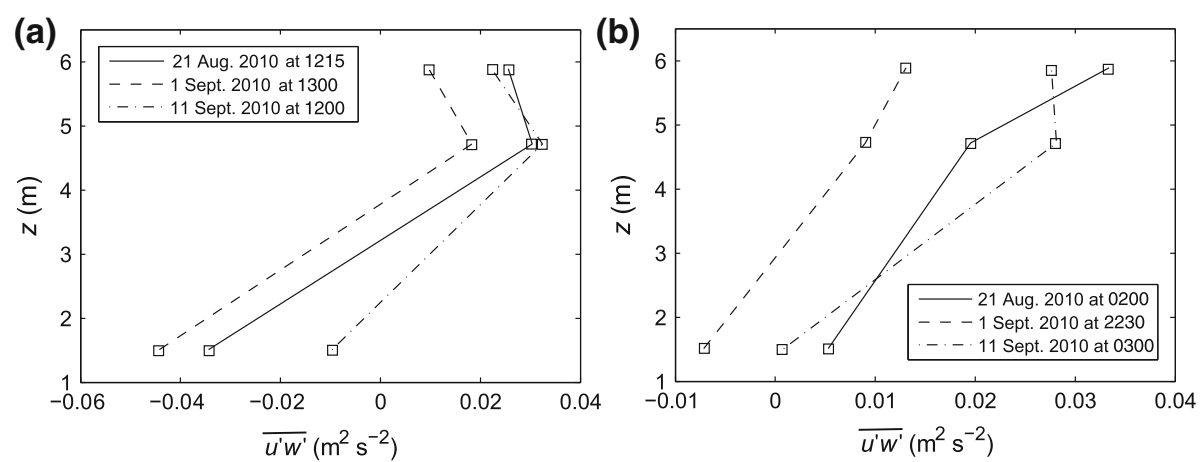

Fig. 6 Sample 15-min momentum flux variations with height for data segments with $\mathbf{a} \zeta<0$ and $\mathbf{b} \zeta>0$ at the lowest observational level of station T2 (1.5 m normal to the surface). Central European times are used

centered finite difference at $3.7 \mathrm{~m}$ is $0.0047 \mathrm{~m} \mathrm{~s}^{-2}$, and the standard deviation is $0.013 \mathrm{~m} \mathrm{~s}^{-2}$. Under stable conditions, these values are respectively 0.0081 and $0.012 \mathrm{~m} \mathrm{~s}^{-2}$. Again, there is not a convincing constant-flux layer, which suggests that MOST is not applicable.

As the turbulence measurements were taken close to the ground $(z \approx 1.5-5.9 \mathrm{~m})$ with a vegetation height of about $0.3 \mathrm{~m}$, it is unlikely that our measurements were above the surface layer, as with other studies using local scaling (e.g. Wood et al. 2010). We argue that the traditional concept of a surface layer is not valid here, perhaps as a result of advection from thermal alpine circulations. Consequently, in the next sections we attempt to scale turbulence characteristics with the local fluxes, instead of the surface fluxes.

\subsection{Dimensionless Standard Deviations of Wind Velocity Components}

In Sects. 4.3-4.5, we evaluate the similarity of scaled standard deviations. Our analysis is focused on the lowest measurement level (1.5 $\mathrm{m}$ above the surface), but results from the two higher observational levels are also reported. To test the functional forms of the nondimensional standard deviations of velocity components (namely (7)—note that the exponent is not a fitting parameter), we used a least-squares analysis. The results are reported in Fig. 7, with a total of 2,413 15-min data segments used. The stabilities ranged from free convective $\left(\zeta_{\min }=-10.2\right)$ to very stable conditions $\left(\zeta_{\max }=37.5\right)$. We chose to plot $\sigma_{u} / u_{* l}$ and $\sigma_{v} / u_{* l}$ since some studies have reported that these scale well with $\zeta$ (e.g. Andreas et al. 1998), and that they should be considered as a MOST statistic (Kader and Yaglom 1990). Note that we assess the self-correlation of these relationships in Sect. 4.6.

The empirical relations we found at $1.5 \mathrm{~m}$ above the surface (at station $\mathrm{T} 2$ ) are:

$$
\begin{gathered}
\frac{\sigma_{u}}{u_{* l}}= \begin{cases}2.84(1-3.61 \zeta)^{1 / 3} & \text { for } \zeta<0, \\
2.85(1+10.55 \zeta)^{1 / 3} & \text { for } \zeta>0,\end{cases} \\
\frac{\sigma_{v}}{u_{* l}}= \begin{cases}2.15(1-3.99 \zeta)^{1 / 3} & \text { for } \zeta<0, \\
2.33(1+5.46 \zeta)^{1 / 3} & \text { for } \zeta>0,\end{cases} \\
\frac{\sigma_{w}}{u_{* l}}= \begin{cases}1.01(1-5.02 \zeta)^{1 / 3} & \text { for } \zeta<0, \\
0.95(1+11.23 \zeta)^{1 / 3} & \text { for } \zeta>0 .\end{cases}
\end{gathered}
$$



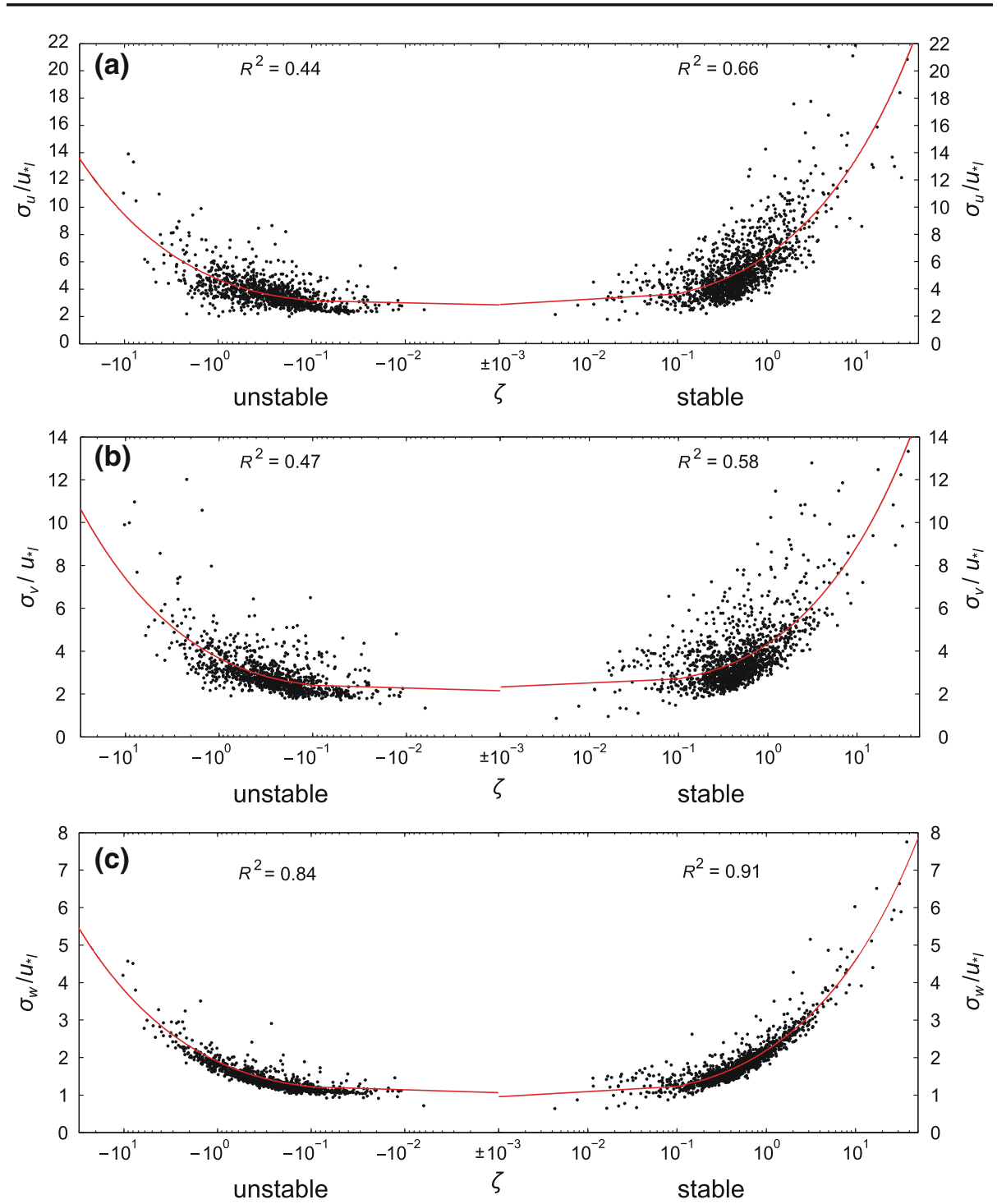

Fig. 7 Locally scaled, dimensionless standard deviations of a streamwise, $\mathbf{b}$ spanwise and $\mathbf{c}$ slope-normal wind velocities at site T2. The measurement level is $1.5 \mathrm{~m}$ normal to the ground. The coefficients of determination for the functional form $\sigma_{i} / u_{* l}=a_{i}\left(1+b_{i} \zeta\right)^{1 / 3}$ are indicated in the top left corner for unstable conditions $(\zeta<0)$, and in the top right corner for stable conditions $(\zeta>0)$

The slope-normal fluctuations of wind-velocity components seem to obey the expected " $1 / 3$ " power law very well. The correlation is much greater than for horizontal velocity variances, and which is likely caused by the nature of the eddies generating the velocity fluctuations. Zhang et al. (2001) argued that the variance of the horizontal wind-velocity components is mostly contained in the low frequencies (large eddies), and that the vertical velocity fluctuations are mostly explained by the high frequencies (small eddies). These small eddies, according to Panofsky and Dutton (1984), are never far from equilibrium since they adapt quickly to variations in the terrain. 
Table 2 Review of near-neutral asymptotic limits of non-dimensionalized velocity fluctuations from studies using the local similarity framework

\begin{tabular}{lllll}
\hline$\sigma_{u} / u_{* l}$ & $\sigma_{v} / u_{* l}$ & $\sigma_{w} / u_{* l}$ & Surface type & References \\
\hline 2.85 & 2.24 & 0.98 & Steep alpine slope & This study (1.5 m, site T2) \\
2.3 & 1.85 & 1.35 & Urban & Wood et al. (2010) \\
2.5 & 2.2 & 1.3 & Urban & Högström et al. (1982) \\
1.76 & 1.6 & 1.22 & Urban & Al-Jiboori et al. (2002) \\
2.35 & 2.1 & 1.35 & Urban & Xu et al. (1997) \\
$\sigma_{V} / u_{* l}{ }^{\mathrm{a}}=2$ & & 1 & Coastal region & Shao and Hacker (1990) \\
\hline
\end{tabular}

The classic values over flat terrain are $\sigma_{u} / u_{*}=2.4, \sigma_{v} / u_{*}=1.9$ and $\sigma_{w} / u_{*}=1.25$ (Garratt 1992)

${ }^{\mathrm{a}} \sigma_{V}=\left(\overline{u^{\prime 2}}+\overline{{v^{\prime 2}}^{2}}\right)^{1 / 2}$

The scatter observed in the horizontal velocity fluctuations (Fig. 7a, b) for the convective regime is in good agreement with numerous other studies (e.g. Krishnan and Kunhikrishnan 2002; Ramana et al. 2004). Panofsky et al. (1977) showed that $z$ and $u_{*}$ are not the appropriate scaling variables for the horizontal velocity fluctuations in the convective surface layer. Indeed, due to low-frequency thermal eddies "sweeping" the bottom of the ABL (Banta 1985; Khanna and Brasseur 1997), the boundary-layer depth $h$ (which constrains the size of these large eddies) and the convective velocity scale $w_{*}$ should be two relevant scaling variables. Unfortunately, given our very sparse measurements of $h$, we could not test the performance of such scaling here. In an alpine valley, the largest turbulent eddies may also be constrained by the valley geometry (de Franceschi et al. 2009).

Surprisingly, the statistical performance is better for stable conditions, possibly due to the poor prediction of (7) in the free convective limit.

In the near-neutral regime $(\zeta \rightarrow 0)$, the non-dimensional variances become independent of $\zeta$ and tend to a constant value. Table 2 presents an overview of near-neutral values published in the literature using a local scaling hypothesis. The values reported in this Table are based on averages of $a_{i}$ in (7) for stable and unstable conditions.

As expected, when $\zeta \rightarrow 0, \sigma_{u} / u_{* l}>\sigma_{v} / u_{* l}>\sigma_{w} / u_{* l}$, highlighting the large anisotropy of the flow. We should note that using local scaling over the city of London, Wood et al. (2010) could obtain similar near-neutral limits of $\sigma_{i} / u_{* l}$ in accordance with those reported over flat and homogeneous terrain where MOST applies. In Table 2, we see that $\sigma_{u} / u_{* l}$ and $\sigma_{v} / u_{* l}$ are slightly above the range of values previously reported in the literature, whereas $\sigma_{w} / u_{* l}$ is much smaller. We hypothesize that this is because our slope coordinate system is not aligned with the gravity vector, hence not fully capturing the turbulence in the vertical direction. Future work should investigate the relationship between slope angles and locallyscaled variances.

In Table 3, we report the statistical performance of similarity relationships at the two highest measurements levels of stations T2. We note that the neutral limits of $\sigma_{u} / u_{* l}$ and $\sigma_{v} / u_{* l}$ decrease with height whereas $\sigma_{w} / u_{* l} \approx 1$ is similar to that found at $1.5 \mathrm{~m}$ above the surface.

\subsection{Dimensionless Standard Deviation of Temperature}

Figure 8 shows the normalized standard deviation of temperature as a function of $\zeta$. In the convective regime, for $\zeta<-0.05$, there is very little scatter and the data follow closely the 
D. F. Nadeau et al.

Table 3 Flux-variance relationships of wind velocities at 4.7 and $5.9 \mathrm{~m}$ above the surface, site $\mathrm{T} 2$

\begin{tabular}{|c|c|c|c|c|c|}
\hline$z(\mathrm{~m})$ & Variable & $\zeta$ & $a_{i}$ & $b_{i}$ & $R^{2}$ \\
\hline \multirow[t]{6}{*}{4.7} & \multirow[t]{2}{*}{$u$} & $<0$ (unstable) & 2.47 & 3.19 & 0.75 \\
\hline & & $>0$ (stable $)$ & 1.59 & 40.61 & 0.74 \\
\hline & \multirow[t]{2}{*}{$v$} & $<0$ (unstable) & 1.88 & 3.37 & 0.85 \\
\hline & & $>0$ (stable $)$ & 1.04 & 68.69 & 0.71 \\
\hline & \multirow[t]{2}{*}{$w$} & $<0$ (unstable) & 1.02 & 4.96 & 0.95 \\
\hline & & $>0$ (stable) & 0.86 & 15.78 & 0.90 \\
\hline \multirow[t]{6}{*}{5.9} & \multirow[t]{2}{*}{$u$} & $<0$ (unstable) & 2.03 & 5.22 & 0.48 \\
\hline & & $>0$ (stable) & 1.90 & 17.82 & 0.53 \\
\hline & \multirow[t]{2}{*}{$v$} & $<0$ (unstable) & 1.54 & 5.83 & 0.46 \\
\hline & & $>0($ stable $)$ & 1.27 & 34.8 & 0.58 \\
\hline & \multirow[t]{2}{*}{$w$} & $<0$ (unstable) & 1.00 & 5.16 & 0.78 \\
\hline & & $>0$ (stable $)$ & 0.99 & 10.3 & 0.77 \\
\hline
\end{tabular}

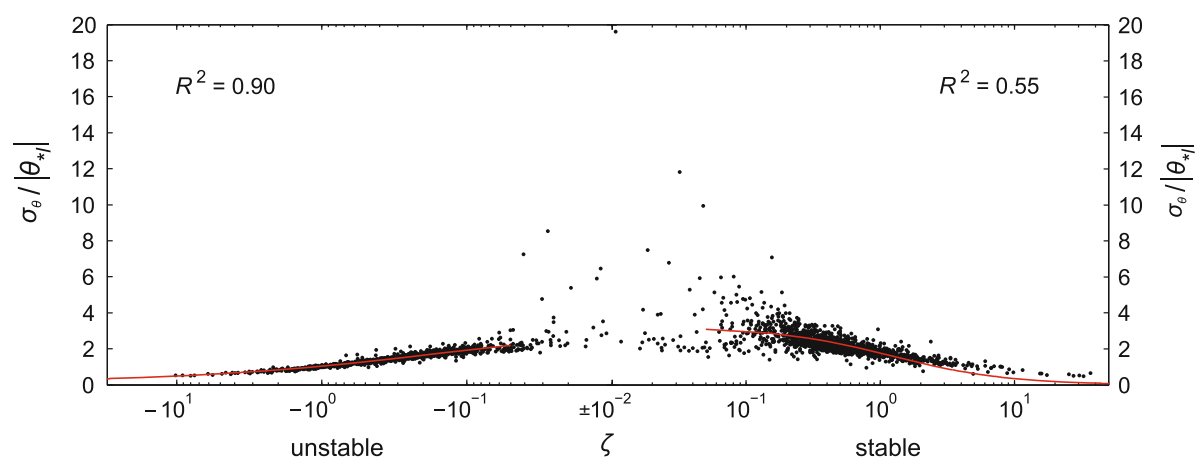

Fig. 8 Locally-scaled, dimensionless standard deviations of virtual temperature fluctuations at site T2. The measurement level is $1.5 \mathrm{~m}$ normal to the ground. The coefficients of determination for the functional form $\sigma_{\theta} /\left|\theta_{* l}\right|=a_{i}\left(1+b_{i} \zeta\right)^{1 / 3}$ are indicated in the top left corner for unstable conditions $(\zeta<0)$. Under stable conditions $(\zeta>0)$, the fitting function is of the form $\sigma_{\theta} /\left|\theta_{* l}\right|=a_{i}\left(1+b_{i} \zeta\right)^{-1}$, and the associated coefficient of determination is specified in the top right corner

“-1/3" scaling. As pointed out by several authors using MOST framework (e.g. Kader and Yaglom 1990; Zhang et al. 2001; Marques et al. 2008), the large scatter for $|\zeta| \rightarrow 0$ is likely caused by the presence of inhomogeneities in air temperature even if the mean buoyancy flux is practically zero. For this reason, the statistical performance is reported exclusively for cases with $|\zeta|>0.05$.

The regression is based on (8) in which the exponents are not free parameters in the curve fit. The fitting curves we found based on a height $1.5 \mathrm{~m}$ above the surface (site T2) are:

$$
\frac{\sigma_{\theta}}{\left|\theta_{* l}\right|}= \begin{cases}2.67(1-16.29 \zeta)^{-1 / 3} & \text { for } \zeta<-0.05 \\ 3.22(1+0.83 \zeta)^{-1} & \text { for } \zeta>0.05\end{cases}
$$

Unlike the velocity fluctuations, the scatter is greater under stable conditions. This is expected given the sporadic nature of nighttime turbulence (Mahrt 1999). The mean ratio $\sigma_{\theta} /\left|\theta_{* l}\right| \approx 2.95$ for near-neutral conditions is within the range of MOST values typically reported in the literature, which vary between 2 and 3 (e.g. Marques et al. 2008). 
Table 4 Flux-variance relationships for temperature at 4.7 and $5.9 \mathrm{~m}$ above the surface, site T2

\begin{tabular}{lllllll}
\hline$z(\mathrm{~m})$ & $\zeta$ & $a_{\theta}$ & $b_{\theta}$ & $c_{\theta}$ & $d_{\theta}$ & $R^{2}$ \\
\hline 4.7 & $<-0.05$ (unstable) & 20.40 & 4,437 & - & - & 0.52 \\
& $>0.05$ (stable) & - & - & 4.29 & 1.86 & 0.30 \\
5.9 & $<-0.05$ (unstable) & 20.29 & 4,457 & - & - & 0.66 \\
& $>0.05$ (stable) & - & - & 3.44 & 0.93 & 0.20 \\
\hline
\end{tabular}

Fig. 9 Locally-scaled, dimensionless standard deviations of specific humidity fluctuations at site $\mathrm{T} 1$. The measurement level is $2.0 \mathrm{~m}$ normal to the ground. The coefficient of determination for the functional form $\sigma_{q} / q_{* l}=a_{i}\left(1+b_{i} \zeta\right)^{-1 / 3}$ is indicated in the top left corner. No data are available under stable conditions

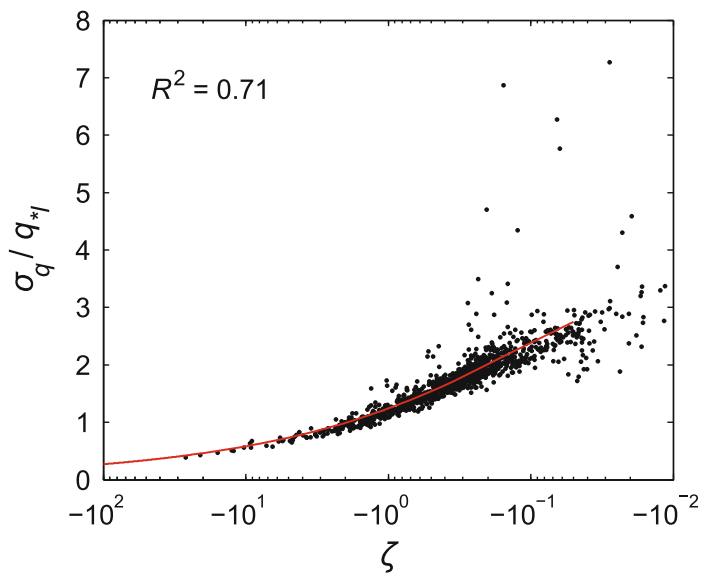

As complementary information, we report the statistical performance of fitting (8) to the turbulence data at the two highest measurement levels (station T2) for the same stability range (Table 4). Large ratios of $\sigma_{\theta} /\left|\theta_{* l}\right|$ are observed for $\zeta \lesssim-0.8$, which explains the unusually large values of $a_{\theta}$ and $b_{\theta}$ in the convective regime.

\subsection{Dimensionless Standard Deviations of Humidity}

Figure 9 shows a plot of $\sigma_{q} / q_{* l}$ versus $\zeta$ from the only point measurements of $q$ collected (site T1, $2 \mathrm{~m}$ above the surface). The orientation of the turbulence sensors at site T1 was such that nighttime (downslope) flow came from behind the instrument. These data runs had to be excluded from the analysis, hence only data runs under convective conditions were analyzed. Cases with a latent heat flux $|L E|<10 \mathrm{~W} \mathrm{~m}^{-2}$ were also discarded to prevent artificially large values of the ratio near the neutral limit. As in Sect. 4.4, given the large scatter for near-neutral conditions, the fit was tested for cases under $\zeta<-0.05$.

Shao and Hacker (1990) reported a greater scatter in $\sigma_{q} / q_{* l}$ than in $\sigma_{\theta} / \theta_{* l}$, and under the MOST framework other investigators have reported similar observations (Tamagawa 1996; Ramana et al. 2004; Zhong et al. 2009). Our observations are consistent with the previous measurements $\left(R_{q}^{2}=0.71\right.$ vs. $\left.R_{\theta}^{2}=0.90\right)$, and the functional form

$$
\frac{\sigma_{q}}{q_{* l}}=3.51(1-21.74 \zeta)^{-1 / 3}
$$


Table 5 Assessment of self-correlation for the flux-variance relationships using the methodology proposed by Klipp and Mahrt (2004)

\begin{tabular}{clllc}
\hline Stability & Variable & $R_{\text {data }}^{2}$ & $\left\langle R_{\text {rand }}^{2}\right\rangle$ & $R_{\text {data }}^{2}-\left\langle R_{\text {rand }}^{2}\right\rangle$ \\
\hline$\zeta<0$ & $u$ & 0.44 & 0.54 & -0.10 \\
& $v$ & 0.47 & 0.50 & -0.03 \\
& $w$ & 0.84 & 0.57 & 0.27 \\
& $\theta$ & 0.90 & 0.32 & 0.58 \\
$\zeta>0$ & $q$ & 0.71 & 0.20 & 0.51 \\
& $u$ & 0.66 & 0.51 & 0.15 \\
& $v$ & 0.58 & 0.41 & 0.17 \\
& $w$ & 0.91 & 0.60 & 0.31 \\
& $\theta$ & 0.55 & 0.57 & -0.02 \\
\hline
\end{tabular}

$R_{\text {data }}^{2}$ is the coefficient of determination for the original data, $\left\langle R_{\text {rand }}^{2}\right\rangle$ is the square of the self-correlation, and $R_{\text {data }}^{2}-\left\langle R_{\text {rand }}^{2}\right\rangle$ is the fraction of variance explained by physical processes. No turbulence data of the specific humidity $q$ are available under stable conditions

for $\zeta<-0.05$, is in good agreement with the observations. Note that, as previously mentioned, the exponent in (13) was taken from (8), and was not a free parameter in the curve fit. In the neutral limit, $\sigma_{q} / q_{* l} \approx 3.51$. Over heterogeneous terrain and under the MOST framework, Andreas et al. (1998) found $\sigma_{q} / q_{*}=4.1$, which is quite close to our value. Overall, the data clearly follow the proposed power-law scaling.

\subsection{Assessment of Self-Correlation}

According to Mahrt (1998), any attempt to evaluate similarity scaling should quantify the role played by self-correlation (see also Hicks 1981). The spurious correlation induced by the presence of $u_{*}$ on both sides of (7) yields a misleading confidence in the applicability of MOST. To quantify the importance of self-correlation, we followed the "random sampling with replacement" approach presented in Klipp and Mahrt (2004). We created 1,000 artificial datasets by randomly redistributing the values of $u_{*}, \overline{w^{\prime} \theta_{v}^{\prime}}, \overline{\theta_{v}}$ and $\sigma_{i}$ with the Matlab ${ }^{\circledR}$ randperm function (sampling without replacement). The self-correlation $\left\langle R_{\text {rand }}\right\rangle$ was defined as the average correlation of these 1,000 datasets. Using this methodology, the difference between the correlation computed for the original dataset $R_{\mathrm{data}}^{2}$ and the randomly generated dataset $\left\langle R_{\text {rand }}^{2}\right\rangle$ is indicative of the fraction of the variance explained by the actual physical processes.

Traditionally, self-correlation has been a concern for the stable boundary layer (e.g. Mahrt 1999). Our results (see Table 5) demonstrate, however, that self-correlation is also significant when the boundary layer is unstable. There appears to be no relationship between the standard deviations of horizontal wind speed and $\zeta$. This confirms that $\Lambda$ and $u_{* l}$ are inappropriate scaling variables for $\sigma_{u}$ and $\sigma_{v}$. For $w, q$ and $\theta$, local scaling is relatively successful, particularly under convective conditions (no data for $q$ when $\zeta>0$ ). Under stable conditions, we expect smaller correlations due to the various transient physical processes peculiar to the nocturnal boundary layer (Cheng et al. 2005). For $\sigma_{\theta}$ this is the case, but for $\sigma_{w}$ the daytime and nighttime actual correlations are surprisingly similar. 

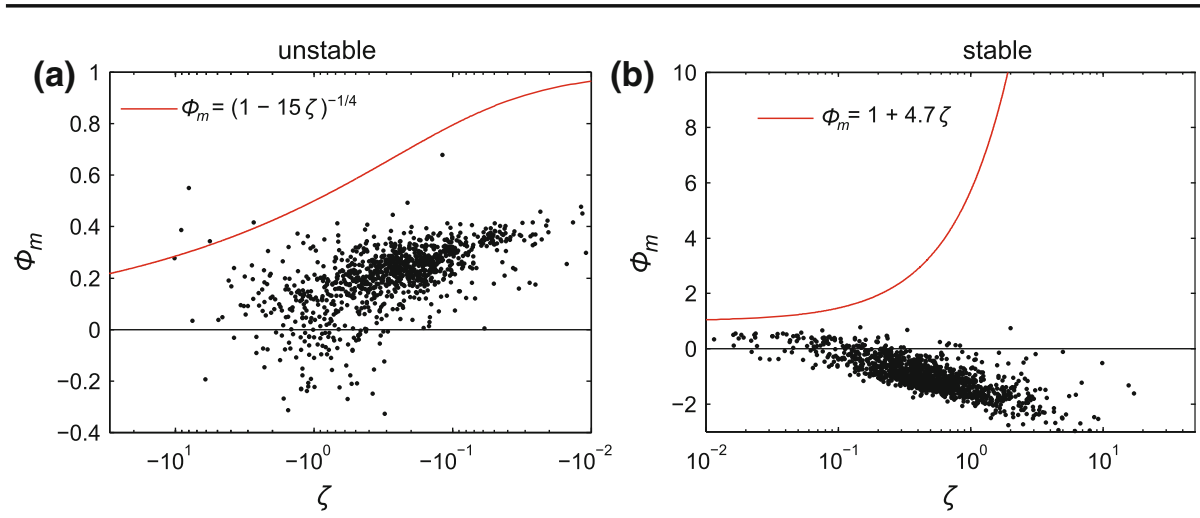

Fig. 10 Dimensionless wind shear $\phi_{m}$ for $\mathbf{a} \zeta<0$ and $\mathbf{b} \zeta>0$ at site T2, $1.5 \mathrm{~m}$ normal to the surface. The solid red lines represent the Businger-Dyer flux-profile relationships determined over flat and homogeneous surfaces (Businger et al. 1971; Dyer 1974)

\subsection{Flux-Profile Relationships}

We have seen in the previous sections that flux-variance relationships could hold along a steep mountain slope, at least for some atmospheric variables and under certain conditions. Using our multi-level measurements, we now investigate the relationship between mean vertical gradients and turbulent fluxes, also known as the flux-profile relationships. All derivatives were evaluated with the forward finite difference method using sonic anemometers from site $\mathrm{T} 2$ at 1.5 and $4.7 \mathrm{~m}$ normal to the surface.

Figure 10 shows the non-dimensionalized wind-speed gradient versus $\zeta$. Given the poor correspondence to the widely accepted Businger-Dyer relationships (Businger et al. 1971; Dyer 1974), we choose not to test fitting functions here. For $\zeta<0$ (Fig. 10a), there are several negative data points, implying that $\partial \bar{u} / \partial z<0$. The amount of data points under the zero line appear too large for all to be caused by instrumental errors. Most of the data points are clustered between 0 and 0.4 , which is much smaller than the classical $\phi_{m}$ function. As discussed earlier, under unstable conditions, Nadeau et al. (2012) argued that the weak mechanical production of turbulence could be due to a valley curvature effect. The combination of an up-valley flow and a sharp turn in the valley axis might lead to an "impinging flow" with very small wind-speed gradients. However further investigations are needed to verify this hypothesis. Overall, the dependence on $\zeta$ appears weak, if not non-existent.

Results for $\zeta>0$ follow those previously described in Sect. 4.3 (Fig. 10b). At night, a very shallow drainage flow (or 'skin flow') forms with a jet close to the ground. At $1.5 \mathrm{~m}$ above the surface, wind speeds decrease with height (Nadeau et al. 2012), leading to $\phi_{m}<0$. Obviously, classical flux-profile relationships are inappropriate to describe nocturnal density currents developing over very steep slopes.

A plot of the dimensionless temperature gradient $\phi_{h}$ versus $\zeta$ is provided in Fig. 11, although the accuracy of temperatures measured by sonic anemometers is limited. Additional temperature profile measurements should be performed to confirm the results presented in Fig. 11, ideally with dense arrays of fine-wire thermocouples. Again, the classical BusingerDyer formulation overpredicts the non-dimensionalized gradient. For unstable conditions, the scattering intensifies when $\zeta>-1$. The measured $\phi_{h}$ appears relatively constant when $\zeta$ varies, except perhaps in the weakly unstable regime when $\phi_{h}$ increases when $\zeta$ decreases. Unfortunately, the scatter in the neutral limit is too large to estimate $\phi_{h}(0)$. 

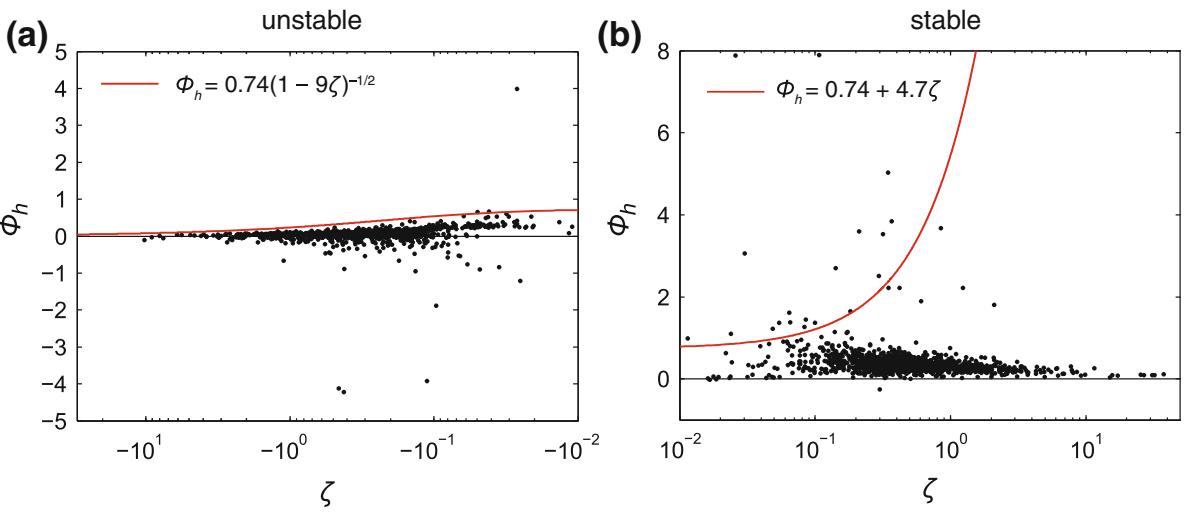

Fig. 11 Dimensionless potential temperature gradient $\phi_{h}$ for $\mathbf{a} \zeta<0$ and $\mathbf{b} \zeta>0$ at site T2, $1.5 \mathrm{~m}$ normal to the surface. The solid red lines represent the Businger-Dyer flux-profile relationships determined over flat and homogeneous surfaces (Businger et al. 1971; Dyer 1974)

Other studies have shown variance similarity to be more successful than gradient similarity. Rannik (1998) reached this conclusion with eddy-covariance data collected in the roughness sublayer over a Scots pine forest in Finland. Similarly, Lenschow et al. (1980) found that the presence of horizontal temperature gradients greatly influence the mean temperature and momentum budgets, while only slightly affecting the second-moment budgets.

Understanding this peculiar behaviour of the stability functions is critical to parametrizing flux transport physics in these types of flows. For example, over flat terrain, Grachev et al. (2007) investigated the effects of self-correlation and stability on the apparent behaviour of the turbulent Prandtl number (a parameter that characterizes the importance of turbulent momentum transfer relative to sensible heat transfer). They found that a bulk Richardson number correlation was more appropriate than other formulations, as it reduced self-correlation. Hence, a reformulation of the flux-gradient methodology in similar terms may be required for slope flows.

\section{Conclusions}

The objective of this study was to test the applicability of similarity scaling over a steep alpine slope. The results were based on eddy-covariance measurements collected during summer 2010 in a narrow valley of the Swiss Alps (slope angles: $30-41^{\circ}$ ). The analysis included convective days when mountain thermal breezes prevail, but also rain-free days when mountain winds were synoptically-driven. It should be noted that we used a slope coordinate system, with the $z$-axis normal to the slope surface.

We first showed that MOST was not applicable due to large variations of the turbulent fluxes with height in the lowest 5-6 m of the atmosphere, where we expected to find a constant-flux layer. On this premise, we tested the validity of local scaling. The results were promising for flux-variance relationships of the slope-normal wind-velocity component, the temperature and specific humidity, and more so under convective conditions. However, after removing the effects of self-correlation, the horizontal velocity fluctuations did not show any relationship with the measurement height and the local Obukhov length. In the neutral limit, we found $\sigma_{u} / u_{* l} \approx 2.85$ and $\sigma_{v} / u_{* l} \approx 2.24$ to be slightly above previously reported values. 
In contrast, $\sigma_{w} / u_{* l} \approx 0.98$ was however smaller, possibly because of our slope coordinate system. Future studies should analyze the locally-scaled variances as a function of slope angle.

The application of flux-profile relationships was not as successful. The observations did not support the classical Businger-Dyer expressions for the non-dimensional profiles of wind speed $\phi_{m}$ and temperature $\phi_{h}$. The discrepancy was greatest under stable conditions, since the observations were collected in the upper region of a shallow drainage flow with $\partial \bar{u} / \partial z<0$, leading to $\phi_{m}<0$. Further work is needed to develop efficient scaling for $\phi_{m}$ and $\phi_{h}$ over sloping terrain.

In essence, our results show that deviations from traditional similarity theory could arise mainly from three factors specific to slope flows: advection from thermal breezes leading to the absence of a constant-flux layer; negative wind-speed gradients due to shallow drainage flows close to the surface; and a misalignment of the coordinate system with the gravity vector. Future field campaigns over steep slopes should include turbulence measurements very close to the slope surface to verify if similarity theory applies in a thin region under the drainage flow wind-speed maximum. Although more in situ observations are needed to confirm our findings, we believe that local scaling can be a useful tool to describe scaled standard deviations in a turbulent alpine boundary layer.

Acknowledgments The authors are grateful to all the collaborators at the Laboratory of Environmental Fluid Mechanics at EPFL who helped with the field campaign, and in particular to Hendrik Huwald. The authors would also like to thank Alain Rousseau from the Institut National de la Recherche Scientifique. This work was funded by the Swiss National Foundation under grant 200021-120238 and by the Office of Naval Research Program Award \# N00014-11-1-0709, Mountain Terrain Atmospheric Modeling and Observations (MATERHORN) Program.

\section{References}

Al-Jiboori MH, Xu YM, Qian YF (2002) Local similarity relationships in the urban boundary layer. BoundaryLayer Meteorol 102:63-82

Andreas EL, Hill RJ, Gosz JR, Moore DI, Otto WD, Sarma AD (1998) Statistics of surface-layer turbulence over terrain with metre-scale heterogeneity. Boundary-Layer Meteorol 86:379-408

Banta RM (1985) Late-morning jump in TKE in the mixed layer over a mountain basin. J Atmos Sci 42:407411

Brooks IM, Rogers DP (2000) Aircraft observations of the mean and turbulent structure of a shallow boundary layer over the Persian Gulf. Boundary-Layer Meteorol 95:189-210

Brutsaert W (2005) Hydrology: an introduction. Cambridge University Press, Cambridge, UK, 605 pp

Brutsaert W, Kustas WP (1987) Surface water vapor and momentum fluxes under unstable conditions from a rugged-complex area. J Atmos Sci 44:421-431

Businger JA, Wyngaard JC, Izumi Y, Bradley EF (1971) Flux-profile relationships in the atmospheric surface layer. J Atmos Sci 28:181-189

Cheng YG, Parlange MB, Brutsaert W (2005) Pathology of Monin-Obukhov similarity in the stable boundary layer. J Geophys Res-Atmos 110:D06101. doi:10.1029/2004jd004923

de Franceschi M, Zardi D, Tagliazucca M, Tampieri F (2009) Analysis of second-order moments in surface layer turbulence in an Alpine valley. Q J R Meteorol Soc 135:1750-1765

Drennan WM, Kahma KK, Donelan MA (1999) On momentum flux and velocity spectra over waves. Boundary-Layer Meteorol 92:489-515

Dyer AJ (1974) A review of flux-profile relationships. Boundary-Layer Meteorol 7:363-372

Foken T (2006) 50 years of the Monin-Obukhov similarity theory. Boundary-Layer Meteorol 119:431-447

Garratt JR (1992) The atmospheric boundary layer. Cambridge University Press, Cambridge, UK, 316 pp

Geissbuhler P, Siegwolf R, Eugster W (2000) Eddy covariance measurements on mountain slopes: the advantage of surface-normal sensor orientation over a vertical set-up. Boundary-Layer Meteorol 96:371-392

Grachev A, Andreas E, Fairall C, Guest P, Persson P (2007) On the turbulent Prandtl number in the stable atmospheric boundary layer. Boundary-Layer Meteorol 125:329-341 
Grisogono B, Kraljevic L, Jericevic A (2007) Notes and correspondence-the low-level katabatic jet height versus Monin-Obukhov height. Q J R Meteorol Soc 133:2133-2136

Grubisic V, Doyle JD, Kuettner J, Mobbs S, Smith RB, Whiteman CD, Dirks R, Czyzyk S, Cohn SA, Vosper S, Weissmann M, Haimov S, De Wekker SFJ, Pan LL, Chow FK (2008) The terrain-induced rotor experiment - a field campaign overview including observational highlights. Bull Am Meteorol Soc 89:1513-1533

Hicks BB (1981) An examination of turbulence statistics in the surface boundary layer. Boundary-Layer Meteorol 21:389-402

Högström U, Bergström H, Alexandersson H (1982) Turbulence characteristics in a near neutrally stratified urban atmosphere. Boundary-Layer Meteorol 23:449-472

Holtslag AAM, Nieuwstadt FTM (1986) Scaling the atmospheric boundary-layer. Boundary-Layer Meteorol 36:201-209

Kader BA, Yaglom AM (1990) Mean fields and fluctuation moments in unstably stratified turbulent boundarylayers. J Fluid Mech 212:637-662

Khanna S, Brasseur JG (1997) Analysis of Monin-Obukhov similarity from large-eddy simulation. J Fluid Mech 345:251-286

Klipp CL, Mahrt L (2004) Flux-gradient relationship, self-correlation and intermittency in the stable boundary layer. Q J R Meteorol Soc 130:2087-2103

Krishnan P, Kunhikrishnan PK (2002) Some characteristics of atmospheric surface layer over a tropical inland region during southwest monsoon period. Atmos Res 62:111-124

Lenschow DH, Wyngaard JC, Pennell WT (1980) Mean-field and 2nd-moment budgets in a baroclinic, convective boundary-layer. J Atmos Sci 37:1313-1326

Lenschow DH, Mann J, Kristensen L (1994) How long is long enough when measuring fluxes and other turbulence statistics. J Atmos Ocean Technol 11:661-673

Mahrt L (1998) Stratified atmospheric boundary layers and breakdown of models. Theor Comput Fluid Dyn 11:263-279

Mahrt L (1999) Stratified atmospheric boundary layers. Boundary-Layer Meteorol 90:375-396

Marques EP, Sa LDA, Karam HA, Alvala RCS, Souza A, Pereira MMR (2008) Atmospheric surface layer characteristics of turbulence above the Pantanal wetland regarding the similarity theory. Agric For Meteorol 148:883-892

Martins CA, Moraes OLL, Acevedo OC, Degrazia GA (2009) Turbulence intensity parameters over a very complex terrain. Boundary-Layer Meteorol 133:35-45

Monin AS, Obukhov AM (1954) Basic laws of turbulent mixing in the ground layer of the atmosphere. Trudy Inst Theor Geofiz An SSSR 151:163-187 in Russian

Nadeau DF, Pardyjak ER, Higgins CW, Huwald H, Parlange MB (2012) Flow during the evening transition over steep alpine slopes. Q J R Meteorol Soc. doi:10.1002/qJ1985

Nieuwstadt FTM (1984) The turbulent structure of the stable, nocturnal boundary-layer. J Atmos Sci 41:22022216

Nordbo A, Järvi L, Haapanala S, Moilanen J, Vesala T (2012) Intra-city variation in urban morphology and turbulence structure in Helsinki, Finland. Boundary-Layer Meteorol. doi:10.1007/s10546-012-9773-Y

Panofsky HA, Dutton JA (1984) Atmospheric turbulence. Models and methods for engineering applications. Wiley, New York, 397 pp

Panofsky H, Tennekes H, Lenschow D, Wyngaard J (1977) The characteristics of turbulent velocity components in the surface layer under convective conditions. Boundary-Layer Meteorol 11:355-361

Park MS, Park SU (2006) Effects of topographical slope angle and atmospheric stratification on surface-layer turbulence. Boundary-Layer Meteorol 118:613-633

Perlik M, Messerli P, Batzing W (2001) Towns in the Alps: urbanization processes, economic structure, and demarcation of European functional urban areas (EFUAs) in the Alps. Mt Res Dev 21:243-252

Ramana MV, Krishnan P, Kunhikrishnan PK (2004) Surface boundary-layer characteristics over a tropical inland station: seasonal features. Boundary-Layer Meteorol 111:153-175

Rannik Ü (1998) On the surface layer similarity at a complex forest site. J Geophys Res-Atmos 103:8685-8697

Rotach MW, Zardi D (2007) On the boundary-layer structure over highly complex terrain: key findings from MAP. Q J R Meteorol Soc 133:937-948

Rotach MW, Andretta M, Calanca P, Weigel AP, Weiss A (2008) Boundary layer characteristics and turbulent exchange mechanisms in highly complex terrain. Acta Geophys 56:194-219

Shao YP, Hacker JM (1990) Local similarity relationships in a horizontally inhomogeneous boundary-layer. Boundary-Layer Meteorol 52:17-40

Sorbjan Z (1988) Local similarity in the convective boundary layer. Boundary-Layer Meteorol 45:237-250

Tamagawa I (1996) Turbulent characteristics and bulk transfer coefficients over the desert in the HEIFE area. Boundary-Layer Meteorol 77:1-20 
Whiteman CD (2000) Mountain meteorology: fundamentals and applications. Oxford University Press, New York, $355 \mathrm{pp}$

Wilczak J, Oncley S, Stage S (2001) Sonic anemometer tilt correction algorithms. Boundary-Layer Meteorol 99:127-150

Wood CR, Lacser A, Barlow JF, Padhra A, Belcher SE, Nemitz E, Helfter C, Famulari D, Grimmond CSB (2010) Turbulent flow at $190 \mathrm{~m}$ height above London during 2006-2008: a climatology and the applicability of similarity theory. Boundary-Layer Meteorol 137:77-96

Xu Y, Chaofu Z, Zhongkai LI, Wei Z (1997) Turbulent structure and local similarity in the tower layer over the Nanjing area. Boundary-Layer Meteorol 82:1-21

Yusup YB, Daud WRW, Zaharim A, Talib MZM (2008) Structure of the atmospheric surface layer over an industrialized equatorial area. Atmos Res 90:70-77

Zhang HS, Chen JY, Park SU (2001) Turbulence structure in unstable conditions over various surfaces. Boundary-Layer Meteorol 100:243-261

Zhong L, Ma Y, Su Z, Lu L, Ma W, Lu Y (2009) Land-atmosphere energy transfer and surface boundary layer characteristics in the Rongbu Valley on the northern slope of Mt. Everest. Arct Antarct Alp Res 41:396-405 